\title{
AiMT
}

Advances in Military Technology

Vol. 15, No. 1, 2020, pp. 115-123

ISSN 1802-2308, eISSN 2533-4123

DOI 10.3849/aimt.01352

\section{Centrifugal Compressor Rate of Stability Assessment}

\author{
A. Jílek* and P. Zelinka \\ Department of Aircraft Technology, University of Defence in Brno, Czech Republic
}

The manuscript was received on 10 September 2019 and was accepted after revision for publication as research paper on 3 March 2020.

\begin{abstract}
:
Small jet and turboprop engines usually use centrifugal compressors. Compressor stable operation is very important for jet engine work and its use. This paper deals with centrifugal compressor stability margin assessment. The appropriate parameter is selected to inform us about working regime in which jet engine (compressor) works in terms of its stable operation.
\end{abstract}

\section{Keywords:}

centrifugal compressor, jet engine, stability margin, standard deviation, surge

\section{Introduction}

Along operation and testing of jet engines, the operating point does not move only across the operating line shown in the compressor characteristics, but also outside of it. In unstable regimes, especially during acceleration, the operating point is reaching close to the surge line.

Compressor surge is one of the unsteady states, which can even escalate and result in the destruction of compressor. Therefore, it is essential to know how dangerous the surge could be. The goal of this study is to find an appropriate parameter that could both analyze and depict this dangerous behaviour.

Each compressor has its own stability margin which is determined by its construction (Fig. 1). To increase the stability margin, several anti-surge devices can be used that are able to move surge line to smaller mass flows in certain regimes of the engine. This means that the compressor could operate stably in a wider range of operation.

The surge is typically preceded by a stall or mild surge causing significant and thus quite easily detectable pressure oscillations. The preceding unstable operation is oscillatory but still quite safe compared to the deep surge. The research described in [1] deals with changes in both the engine slow pressures and fast parameters (pres-

\footnotetext{
* Corresponding author: Department of Aircraft Technology, Faculty of Military Technology, University of Defence in Brno, Kounicova 65, CZ-662 10 Brno, Czech Republic. Phone: +420973443366,E-mail: adolf.jilek@unob.cz
} 
sures, noise, vibrations). The goal of this research was to describe typical changes in engine parameters and to define a stipulated limit of engine safe operation.

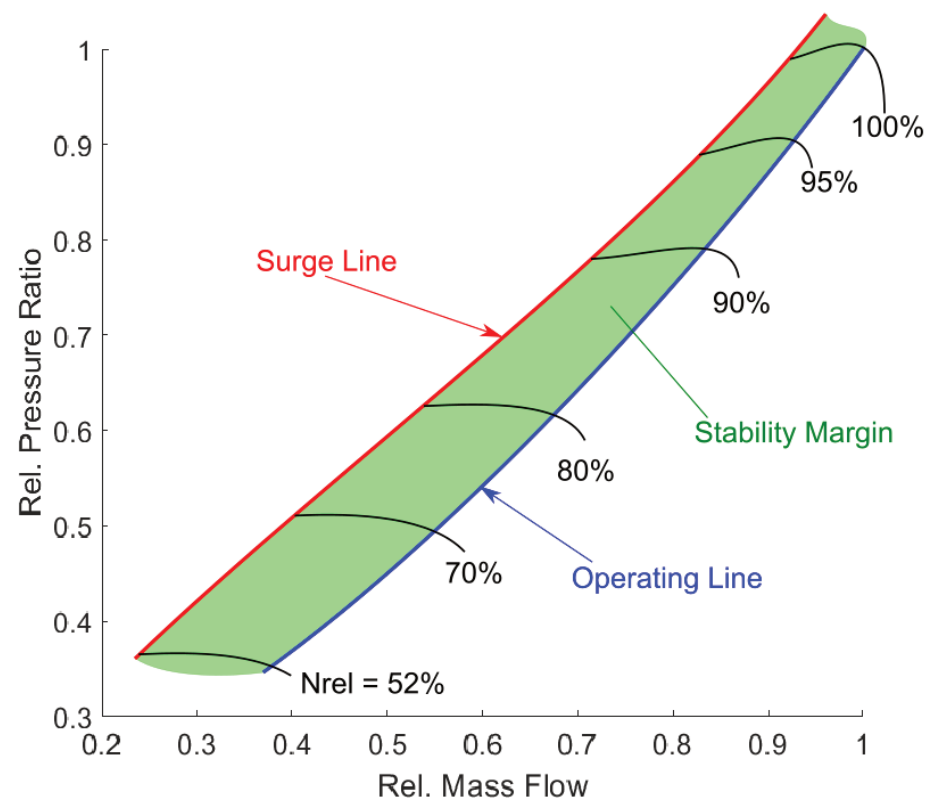

Fig. 1 Compressor characteristics

\section{Measurement Description}

Experiments were conducted utilizing the small jet engine TJ100 with variable exhaust nozzle at the University of Defence in Brno. The engine design pressure ratio is approximately 4.5 , and it is rated to maximum thrust of $1 \mathrm{kN}$ at rotational speed of approximately $60000 \mathrm{rev} / \mathrm{min}$. The engine has a single stage centrifugal compressor with radial and axial bladed diffusers, reversed flow combustion chamber and a single stage uncooled axial turbine. Engine layout together with all measurement parameters and their respective positions are depicted in Fig. 2.

Current diagnostic system is an evolution of the design described in [1] and it is quite similar to the system described by Fözö [2]. In the test, combination of "slow" pressure transmitters, flush mounted high bandwidth pressure probes, accelerometers and a microphone were used. The measurements were performed at three different sampling rates. $50 \mathrm{~Hz}$ rate was used for temperature measurements (labelled black in Fig. 2) and for the evaluation of steady state thermodynamic parameters. Temperatures were measured by thermocouples and engine speed was evaluated at this rate from a pulse signal read from the engine control system. The 'slow' pressures (blue) were measured by BD SENSORS digital pressure transmitters which incorporate A/D convertor working at $5 \mathrm{kHz}$ sampling rate. Finally, $50 \mathrm{kHz}$ rate (red) was used for acoustic noise, vibrations (in three directions $\mathrm{X}, \mathrm{Y}$ and $\mathrm{Z}$ ) and Kulite "fast" pressure transmitters. Engine parameters were measured along the steady state operating line in the range from idle $(50 \%)$ to $95 \%$ of $\max R P M$ with fixed exhaust nozzle. Subsequently, constant speed lines were evaluated by throttling the engine with a variable exhaust 
nozzle. The compressor map was built from the $50 \mathrm{~Hz}$ data. Steady state parameters at each point of the map were calculated as average from 1-second-long data waveform.

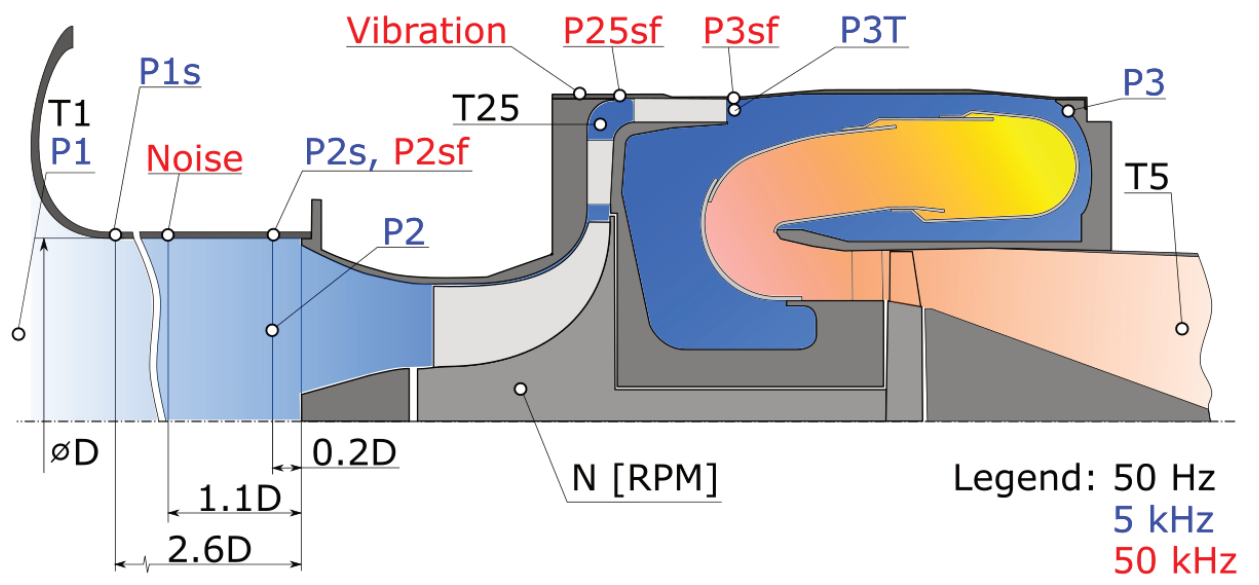

Fig. 2 Scheme of small jet engine test rig with indication of measured parameters

The characteristics of the compressor have been repeatedly measured. It includes determining jet engine operating line and speed lines for different $R P M$. The data from $[3,4]$ was also used and then analyzed.

\section{Selection of the Evaluated Value}

To detect the compressor unsteady work, it is necessary to find parameters which show the increase of instability.

Common approaches to detect compressor unsteady work are:

- the change of the temperature - an approaching surge can be detected by the thermocouples located in the inlet duct in front of the compressor impeller. The thermocouples are situated in different positions in the inlet duct. When the mass flow rate at $N_{\text {rel }}=$ const. decreases, (near surge line) the thermocouples closest to impeller indicate significant increase in temperature gradient. This demonstrably determines the approaching surge,

- the change of the pressure - another suitable parameter for detecting instability is the pressure pulsation. This occurs more or less in all parts of the centrifugal compressor. But question is which pressure shows the most relevant value. When the mass flow rate decreases at constant RPM, the pressure patterns will change. Fig. 3 shows the initialization of surge by pressure oscillations (A - stable, B - mild surge, C - surge),

- the change of inlet velocity field - the upcoming instability is detectable by the change of the velocity in the inlet duct (in front of the impeller). If the compressor work is stable, the velocity field in front of the impeller is relatively uniform and no negative velocities appear near the impeller inlet.

There are other ways how to detect unstable compressor work, which are based on evaluating other different variables. Instability can be reliably proven by noise measurement using microphones. Another indicator may also be the engine vibration analysis, when the instability causes an increasing trend of intensity of these vibrations [1]. 


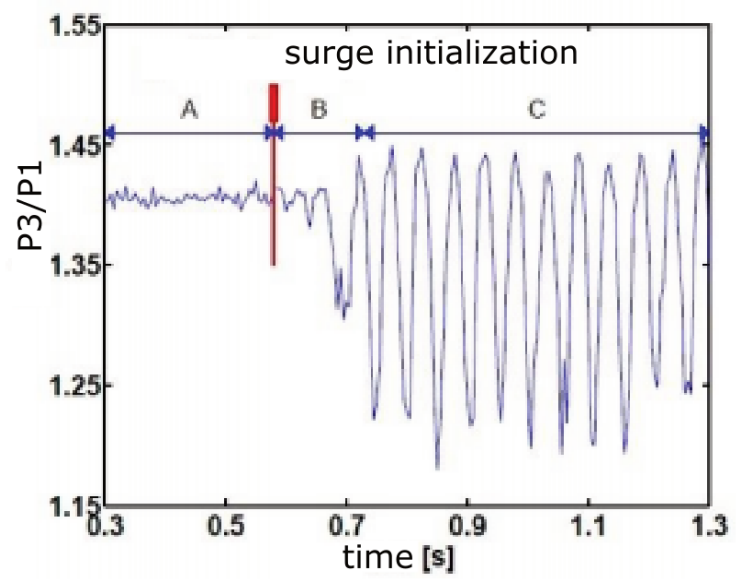

Fig. 3 Pressure oscillations when surge begin [5]

During the engine operation or when performing experiments in the test cell, it is more convenient to use some standard variable that is commonly measured for other purposes of engine control to detect the engine instability. Previous research [1] has shown that it is not necessary to use "fast" pressure sensors, or vibrations. "Slow" pressures measured behind the compressor are sufficient and can provide clearer information about engine regime.

The $P 3$ pressure was selected for stability evaluating. There are two positions where the pressure $P 3$ was measured - one was at the beginning of the combustion chamber $(P 3 T)$ and the other one was on the back wall (P3). An analysis showed that both sensors produced similar signals. The second one - on the back wall of combustion chamber - is used by the producer for pressure ratio evaluation and that is why it was selected (Fig. 4).

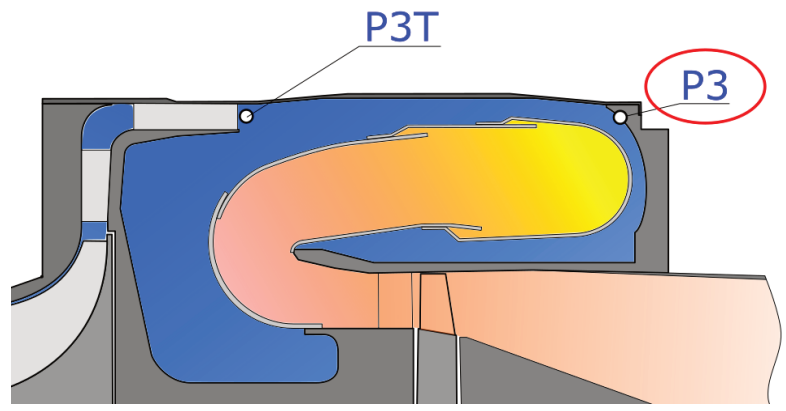

Fig. 4 P3 pressure sensors

$P 3$ pressure oscillations were the best detectable at every speed lines and in the full range of mass flow rate. The $5 \mathrm{kHz}$ sampling rate provides enough information about the work of the engine and it is not as demanding on hardware as $50 \mathrm{kHz}$ sampling rate. 


\section{Selection of the Evaluated Parameter}

Measurement of $P 3$ pressure was performed by the BD SENSORS transmitters and data acquisition was done with cRIO computer by NI. The recorded and analyzed signals were $1 \mathrm{~s}$ long. Analysis relied on these parameters of descriptive statistics (Fig. 5):

- Standard Deviation (StDev),

- Crest Factor (PEAK2RMS),

- Kurtosis Factor.

Analysis shows that sufficient information about compressor behaviour dives the standard deviation of the measured values. In addition, the software requirements are relatively low which a great advantage is. It is possible to evaluate the standard deviation directly via measurement without a great influence on the real-time processing of further values.
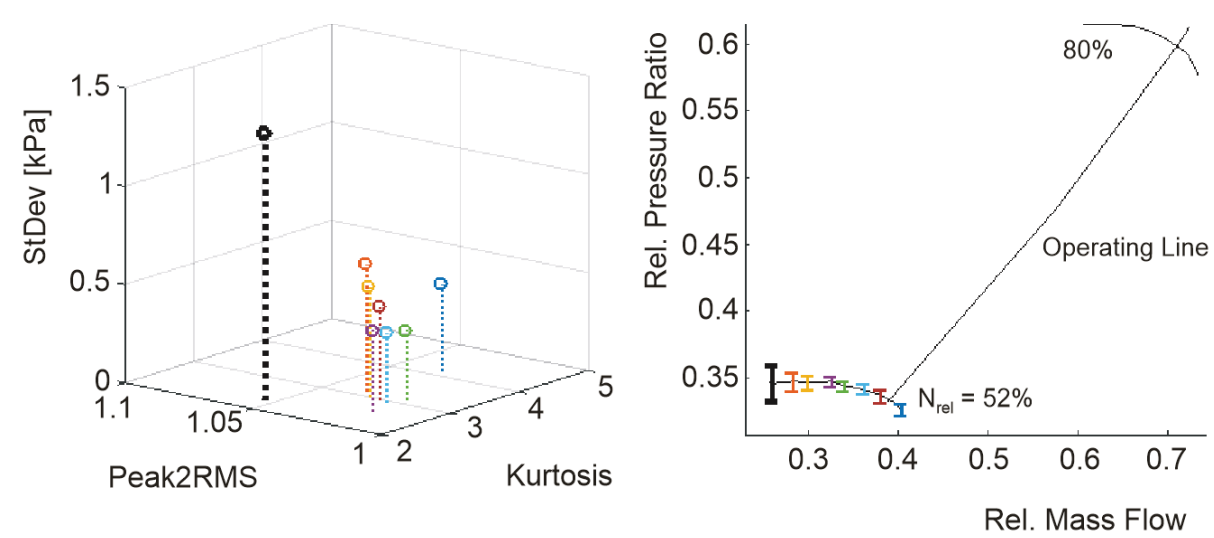

Fig. 5 Parameters of descriptive statistics used for speed line $N_{\mathrm{rel}}=52 \%$ [1]

The standard deviation was estimated based on the values obtained during the compressor characteristic measurement (Fig. 6). The measured points and respective air mass flow rate standard deviations are displayed as vertical bars. The difference between two consecutive points is approximately $1 \%$ of the air flow rate. It is clear that when mass flow rate decreases at $N_{\text {rel }}=$ const. and measured point approaches to surge line, standard deviation of $P 3$ pressure increases.

The change of the StDev has also been reflected in the change of RPM. The standard deviation increases with rotation speed. This means that the same change in the standard deviation of pressure $P 3$ can be caused by a change of $R P M$ without the working point approaching the surge line. Theoretically, compressor operates most stably at the steady state operating line which corresponds to different standard deviations (Fig. 7).

Relative standard deviation (RSD) was introduced for the evaluation of the stability assesment. It is the ratio of the standard deviation of $P 3$ pressure to the standard deviation measured at the engine operating line for the same RPM.

$$
R S D=\frac{S t D e v}{S t D e v_{O L}}
$$




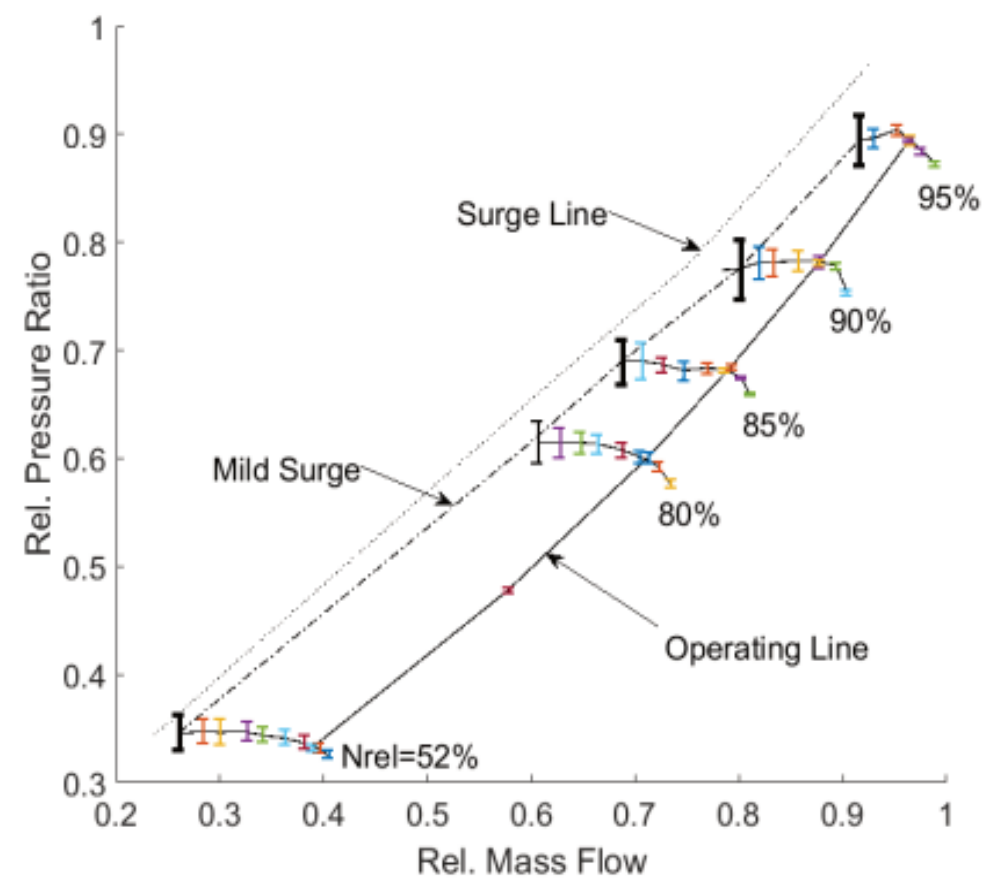

Fig. 6 Compressor map [1]

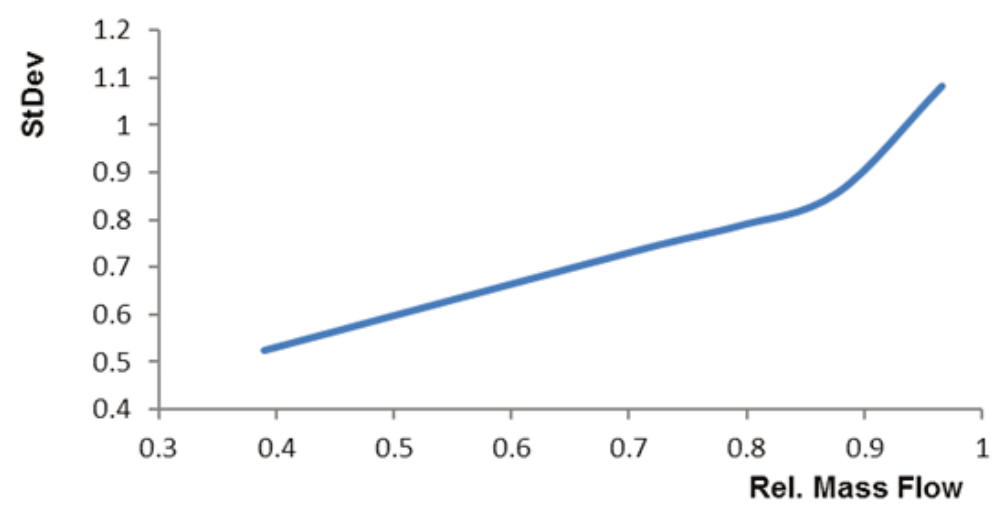

Fig. 7 The change of standard deviation for different RPM on operating line

This parameter considers different oscillation of the $P 3$ pressure for different speed lines. Similary, relative mass flow rate $W R$ was introduced as the ratio of the mass flow rate of measured point to the mass flow rate at the engine operating line for the same RPM.

$$
W R=\frac{\text { RelMassFlow }}{\text { RelMassFlow }_{O L}}
$$

The following pictures show the relative standard deviations at $80 \%$ and 90\% RPM (Fig. 8). 
The graphs show (mainly at $80 \% R P M$ ) an increase in instability not only when the mass flow rate decreases, but also when the compressor operates at a higher mass flow rate than on the operating line $(W R=1)$.

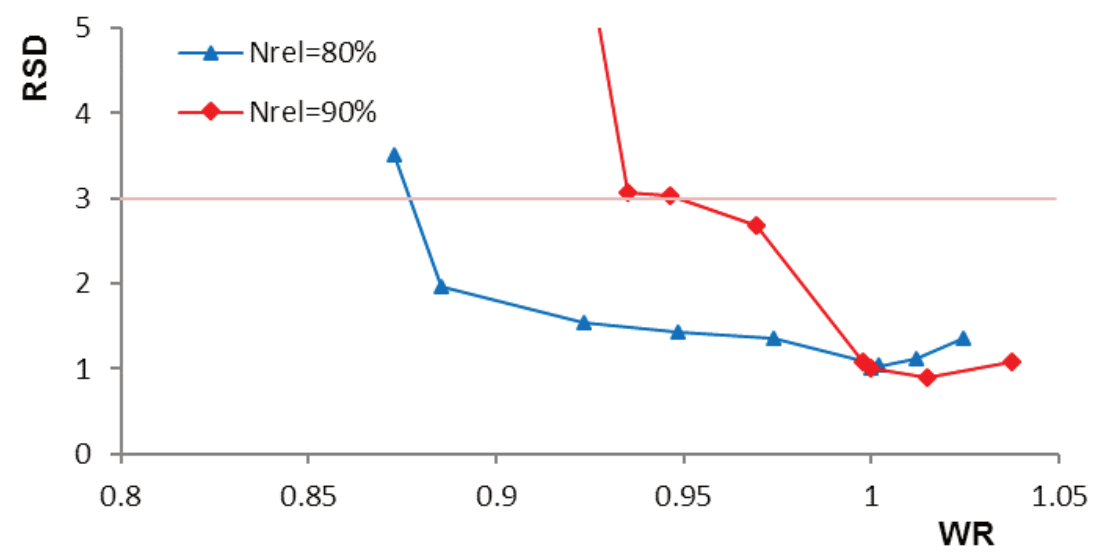

Fig. 8 Relative standard deviations at $80 \%$ and $90 \%$ RPM

\section{Establishment of Critical Value}

Once the relative standard deviation has been analyzed, it is necessary to determinate the critical value. The value of the relative standard deviation may reach different values (Fig. 8). If the operator decreases the mass flow rate too much at the constant $R P M$, the $R S D$ value approaches 9 . In this case, the $P 3$ pressure oscillates a lot and the compressor work is unstable (Fig. 9).

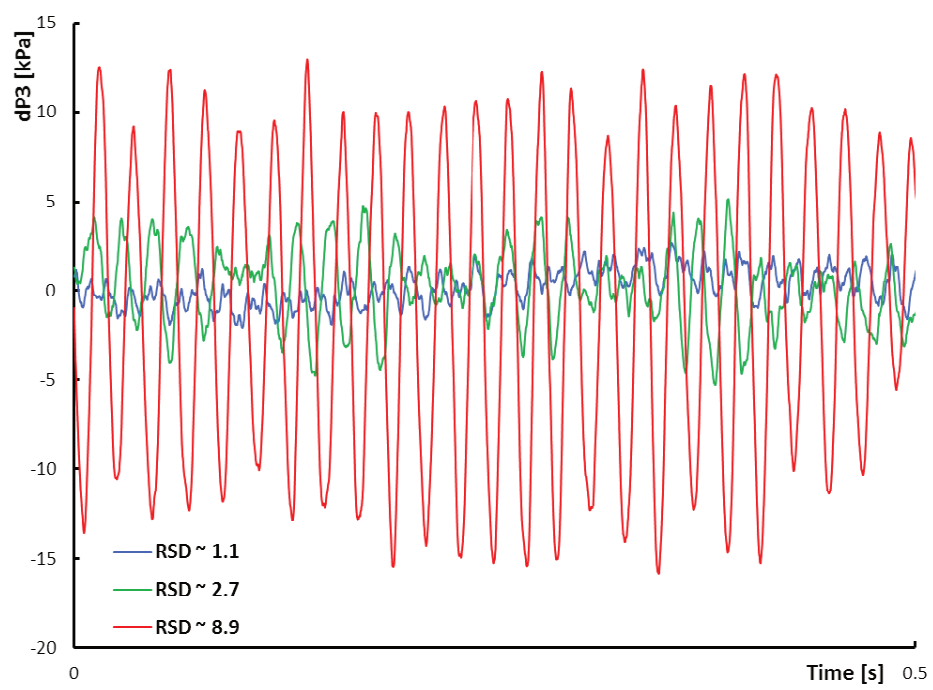

Fig. 9 P3 pressure oscillation for various relative standard deviation at $90 \%$ RPM 
When the operating point is in close proximity to the operating line, the pressure oscillation is small and the relative standard deviation is close to 1 (blue curve, Fig. 9). As the relative standard deviation exceeds value 3 , significant irregular pressure oscillations can be observed (Fig. 10). The amplitude of pressure P3 is not constant, but varies within a relatively wide range. These oscillations correspond to the socalled mild surge.

This analysis was performed for all measured RPMs and the courses were similar. Based on our measurements, the value of $R S D$ of 3 was chosen as critical, as the beginning of mild surge.

The jet engine can be operated beyond the mild surge line without the risk of damage (for a short time during acceleration). However, pressures and other parameters (temperatures, ...) oscillate and it is not desirable to operate the engine in these regimes for a long time.

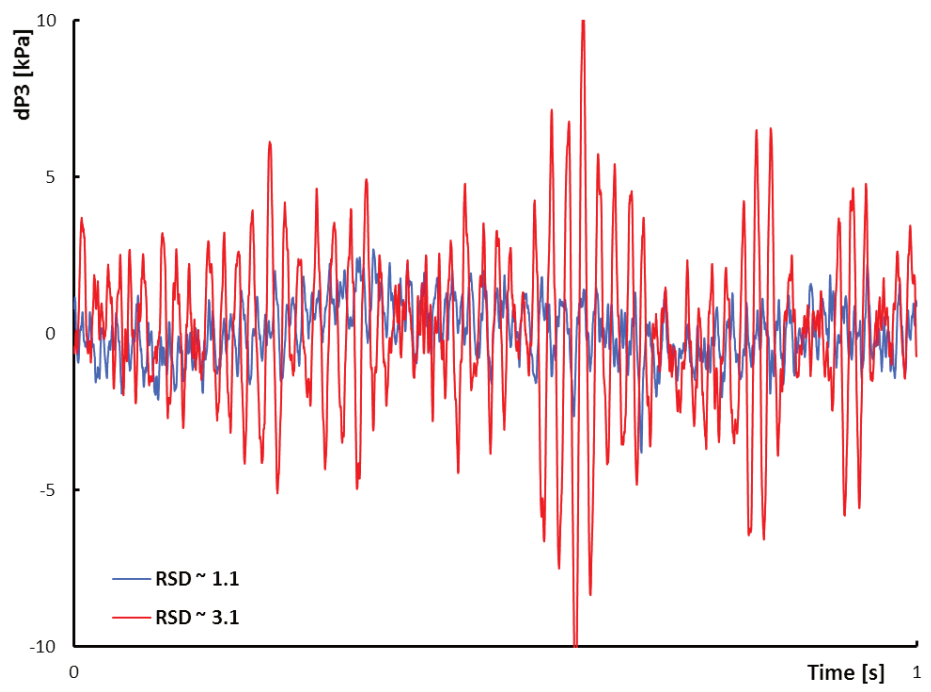

Fig. 10 P3 pressure oscillation at the mild surge deviation at $90 \%$ RPM

\section{Conclusion}

The problem of stable compressor work is very broad and has been the subject of research teams for a long time. The aim of the research was to find a suitable parameter that would reliably inform us of the approaching compressor surge. Several parameters and quantities have been explored at different sampling rates. The analysis performed proved to be the most appropriate use of $5 \mathrm{kHz}$ sampling pressure in the combustion chamber. $R S D$ of $P 3$ was selected as an evaluation criterion. By means of relative standard deviation, the mild surge was established. The critical value of the relative standard deviation indicating the mild surge was determined by analyzing the pressure oscillations during the entire speed range.

The rate of stability is important to the operator who performs the measurement on the compressor or on the jet engine. It can also be used to check jet engines to prevent unstable work. 
At present, the stability rate information is linked to the oscillation of pressure at the operating line for a particular $R P M$. The values of the standard deviation of the pressure oscillation on the measured $R P M$ point to a trend, which can be used to asses the stability of the compressor on yet unmeasured $R P M$. This could lead to further research to determine another parameter, which would allow to determine the compressor stability beyond the previously measured speed.

\section{Acknowledgement}

This work was funded by the Ministry of Defence of the Czech Republic under the framework of University of Defence in Brno development program - DZRO K205. Research was supported by the manufacturer of the tested small jet engine TJ $100-$ PBS Velká Bíteš, a.s.

\section{References}

[1] PEČINKA, J., JÍLEK, A. and KMOCH, P. Small Jet Engine Centrifugal Compressor Stability Margin Assessment. In Proceedings of the ASME Turbo Expo 2017: Turbomachinery Technical Conference and Exposition. Charlotte: ASME, 2017. ISBN 978-0-7918-5081-7.

[2] ANDOGA, R., FÖZÖ, L., MADARÁSZ, L. and KAROL, T. A Digital Diagnostic System for a Small Turbojet Engine. Acta Polytechnica Hungarica, 2013, vol. 10, no. 4, p. 45-58. ISSN 1785-8860.

[3] JÍLEK, A., PEČINKA, J. and SAMPATH, S. Compressor Fouling Influence on High Frequency Data Signals. In Proceedings of the $23^{\text {rd }}$ International Symposium on Air Breathing Engines (ISABE 2017). New York: ISABE, 2017, vol. 3/4, p. 2017-2028. ISBN 978-1-5108-7279-0.

[4] PEČINKA, J., GBANAIBOLOU, J., SAMPATH, S. and DAVID, M. Influence of Fouling on Compressor Dynamics: Experimental and Modeling Approach. Journal of Engineering for Gas Turbines and Power, 2018, vol. 140, no. 3, paper no. GTP-17-1202. DOI 10.1115/1.4037913.

[5] MEULEMAN, C.H.J. Measurement and Unsteady Flow Modelling of Centrifugal Compressor Surge. Eindhoven: Technische Universiteit Eindhoven, 2002. 146 p. DOI 10.6100/IR557873. 